\title{
Precise microstructuring of indium-tin oxide thin films on glass by selective femtosecond laser ablation
}

\author{
S. Krause ${ }^{1}$, P.T. Miclea ${ }^{1,2}$, F. Steudel ${ }^{1}$, S. Schweizer ${ }^{1,3}$, and G. Seifert ${ }^{1,4, a}$ \\ 1 Fraunhofer Center for Silicon Photovoltaics CSP, Walter-Hülse-Str. 1, 06120 Halle (Saale), Germany \\ 2 Institute of Physics, Martin Luther University of Halle-Wittenberg, Heinrich-Damerow-Str. 4, 06120 Halle (Saale), Germany \\ 3 Department of Electrical Engineering, South Westphalia University of Applied Sciences, Lübecker Ring 2, \\ 59494 Soest, Germany \\ ${ }^{4}$ Centre for Innovation Competence SiLi-nano ${ }^{\circledR}$, Martin Luther University of Halle-Wittenberg, \\ Karl-Freiherr-von-Fritsch-Str. 3, 06120 Halle (Saale), Germany
}

Received: 11 October 2012 / Received in final form: 4 December 2012

Published online: 4 February 2013

(C) Krause et al., published by EDP Sciences, 2013

\begin{abstract}
Transparent conductive oxide (TCO) thin films were removed from glass substrates using femtosecond laser pulses. Irradiating through the glass, the threshold for complete TCO ablation was much lower than for front-side irradiation. Additionally, the former method created almost rectangular crosssectional groove profiles despite the Gaussian laser beam. This indicates a non-thermal ultrafast ablation mechanism via critical carrier concentration achieved by the femtosecond pulse in the TCO at the interface. Very narrow scribes of only $5 \mu \mathrm{m}$ width provided very good electrical separation, making this technique very attractive for micro-structuring applications like scribing of thin-film solar cells.
\end{abstract}

\section{Introduction}

Transparent electrodes consisting of conductive oxide thin films on flat glass are nowadays widely used as basis of technical applications like flat panel displays or solar cells. Traditionally, patterning of such transparent conductive oxide (TCO) films has been done by lithography. However, laser ablation techniques are becoming more and more attractive because they offer - instead of several processing steps including chemical wet etching - a maskless, onestep, dry process which can be conducted under ambient conditions. To comply with the need of precise microstructures like, for instance, groove widths below $10 \mu \mathrm{m}$ providing perfect electrical isolation, ultrashort pulses have to be used in order to avoid any thermal damage or contamination in the ablated region. Previous empirical studies using pico- or femtosecond pulses at several wavelengths from the near infrared to ultraviolet spectral range showed that a fairly "clean" ablation of TCOs or photovoltaic absorber thin films is possible by irradiating successive pulses with considerable spatial overlap from the TCO side [1-7].

These investigations, however, did not consider in detail the physical mechanism of the TCO ablation. Very recent results reported by Rublack et al. [8,9] suggest an interesting approach for fs pulses: it was shown that dielectric coatings can be removed selectively from silicon wafers

${ }^{a}$ e-mail: gerhard.seifert@physik.uni-halle.de by fs laser ablation in such a way that the opened surface is perfectly smooth and the Si material below the ablated area is still perfectly crystalline. The key point here is free carrier absorption in the semiconductor created by the rising edge of the fs pulse itself, which leads to non-thermal decomposition of a very thin silicon layer of a few nanometers thickness only, the expansion of which blasts off the capping dielectric layer. Transferring this idea to the reverse case of TCO on glass (conductive layer on dielectric substrate), a similar ablation mechanism should be possible for irradiating fs pulses through the glass, thereby creating free carrier absorption in the TCO at the interface. So far, ablation experiments using rear-side irradiation with fs pulses have only been reported for metals like chromium [10], but not for TCO.

In this work, it is demonstrated that this approach really enables a damage-free (with respect to substrate and remaining film) ablation of TCO films. For this purpose, the ablation of indium-tin oxide (ITO) films on glass by $300 \mathrm{fs}$ pulses at $1030 \mathrm{~nm}$ was studied; the laser irradiation was carried out from the glass as well as from the ITO side.

\section{Experimental}

The samples studied in this work were approximately $600 \mathrm{~nm}$ thick ITO layers on soda-lime float glass sheets 


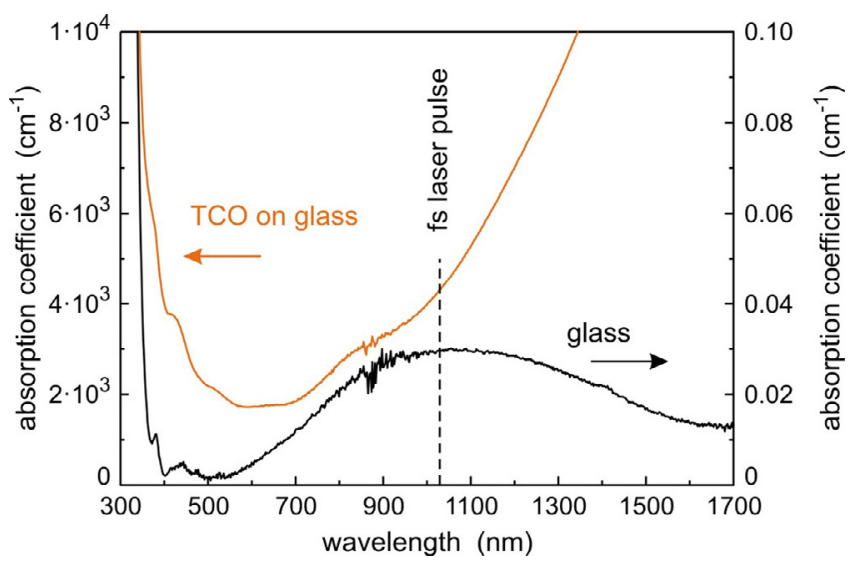

Fig. 1. Absorbance of an ITO/glass sample (orange curve) and a pure glass substrate (black curve); the laser wavelength is indicated by a vertical dashed line.

of approximately $3 \mathrm{~mm}$ thickness. Typically, such glasses are the base material for the production of inorganic thinfilm solar cells. For the laser structuring of the ITO layer a femtosecond Yb:KGW laser system (PHAROS, LightConversion) was used with a wavelength of $1030 \mathrm{~nm}$ and pulse duration of $300 \mathrm{fs}$, average power of up to $6 \mathrm{~W}$ and maximum repetition rate of $350 \mathrm{kHz}$. These laser pulses were focused to the sample surface with an $f$-theta lens having a focal length of about $55 \mathrm{~mm}$ (yielding a nearly Gaussian beam of $13.0 \pm 0.5 \mu \mathrm{m}$ diameter at the beam waist), in connection with an $x y z$-scanner for positioning and motion control.

In Figure 1, the absorbance spectra (obtained from total transmittance and reflectance measured with an integrating sphere) of the ITO/glass samples and the bare glass substrate are compared. Considering the vastly different thickness of thin film and substrate, these data indicate a fairly large absorption coefficient of $\alpha \approx 4000 \mathrm{~cm}^{-1}$ for the ITO layer at the laser wavelength of $1030 \mathrm{~nm}$, but a comparably negligible one for the glass substrate $\left(\alpha \approx 0.03 \mathrm{~cm}^{-1}\right)$. Due to this choice, experiments could be performed irradiating the samples from the ITO side as well as from the rear side, i.e. with the laser beam travelling through the glass before interacting with the ITO film. For comparison, also pure glass samples were irradiated in the same way. By varying the scribing speed, single ablation spots as well as lines consisting of successive pulses with well-defined overlap were produced. To be able to vary the pulse energies in a precise and reproducible way, an attenuator consisting of a half-wave plate and a thinfilm polarizer were used. The actual pulse energies were then determined by measuring the average power in front of the samples with a thermopile detector, considering the chosen repetition frequency. Ablation thresholds were obtained in analogy to Liu's method [11] by evaluating the size of the ablated areas as a function of laser pulse energy (fluence), as described in detail previously [8].

After laser processing, the ablation spots or lines were characterized using optical microscopy (images were taken with a Leica DM RXE-650H microscope) and profilometry (cross-sectional profiles were taken with an Ambios Technology profilometer XP-2). Scanning electron microscopy (SEM) images were acquired with a JSM-7401F (JEOL) electron microscope.

\section{Results and discussion}

In a first series of experiments, various "scribes" were made at different laser fluences, with a pulse-to-pulse distance of $7 \mu \mathrm{m}(0.35 \mathrm{~m} / \mathrm{s}$ at $50 \mathrm{kHz}$ pulse repetition frequency); irradiation was carried out from the top (ITO surface) and from the rear side (through the glass substrate).

Figure 2 shows a selection of results in form of microscope images (Figs. 2a and 2b) and the corresponding cross-sectional profiles obtained at positions close to the spot centers of an arbitrary laser shot within the scribe. Figures $2 \mathrm{a}$ and $2 \mathrm{c}$ refer to irradiation from the TCO side, Figures $2 \mathrm{~b}$ and $2 \mathrm{~d}$ to the laser beam coming from the glass side. Discussing first the results in Figures 2a and 2c in the sequence of increasing fluence, one recognizes as main trend the creation of ablated regions of increasing maximum depth, $d$, and width, $b$, showing a cross-sectional profile which is more or less similar to the Gaussian laser beam profile. Starting with $b \approx 5 \mu \mathrm{m}$ and $d \approx 250 \mathrm{~nm}$ at $1.5 \mathrm{~J} / \mathrm{cm}^{2}$, a value of $d \approx 600 \mathrm{~nm}$ corresponding to the total ITO layer thickness is only achieved at $12.3 \mathrm{~J} / \mathrm{cm}^{2}$. At $18.4 \mathrm{~J} / \mathrm{cm}^{2}$, the groove is $b \approx 13 \mu \mathrm{m}$ wide and $d \approx 650 \mathrm{~nm}$ deep; apparently, as indicated by the highest fluence result, the ablation does not stop at the former ITO/glass interface. Looking in more detail, the intermediate fluences exhibit a comparably steep hole in the crater centers, while the second lowest one even exhibits a small bulge there. Additionally, for the lowest fluence, the main deep hole seems to be surrounded by a shallow one of only a few nanometers of ITO material having been removed.

The results of irradiation through the glass exhibit a quite different behavior: first, ablation starts at considerably lower laser energy density; second, even at the lowest fluence the ablation depth $d \approx 600 \mathrm{~nm}$ indicates complete removal of the ITO layer; third, the ablation crosssectional profile looks almost rectangular in any case, with steep edges and almost flat bottom. Looking in detail, a 20-30 nm deep dimple towards the center of ablation can be observed in particular for the higher laser energies.

The rear-side irradiation, i.e. irradiation from the glass side, is similar to recent results described by Rublack et al. $[8,9]$, who identified the fs laser induced non-thermal evaporation of an ultrathin $(\leqslant 5 \mathrm{~nm})$ Si layer as the key process for selective ablation of $\mathrm{SiO}_{2}$ or $\mathrm{Si}_{x} \mathrm{~N}_{y}$ layers from silicon wafers without detectable damage to the substrate. If a similar, nonlinear absorption process occurs in ITO, already fluences slightly below the actual ablation threshold should cause a separation of the ITO layer from the substrate and a corresponding bulge. In fact, already at $0.6 \mathrm{~J} / \mathrm{cm}^{2}$, a shallow wall of $30-70 \mathrm{~nm}$ height is found instead of a $600 \mathrm{~nm}$ deep groove. A cross section through such a bulge is shown in the scanning electron microscopy (SEM) image of Figure 3, where the $\approx 600 \mathrm{~nm}$ thick ITO 
(a)

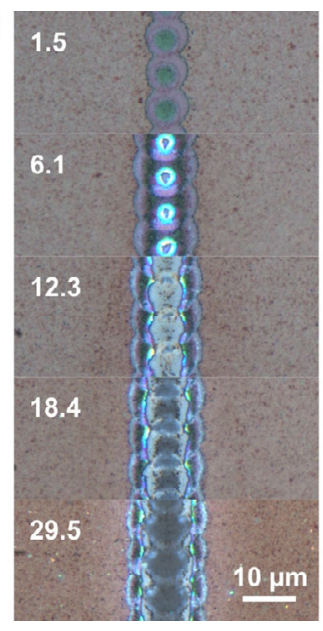

(b)
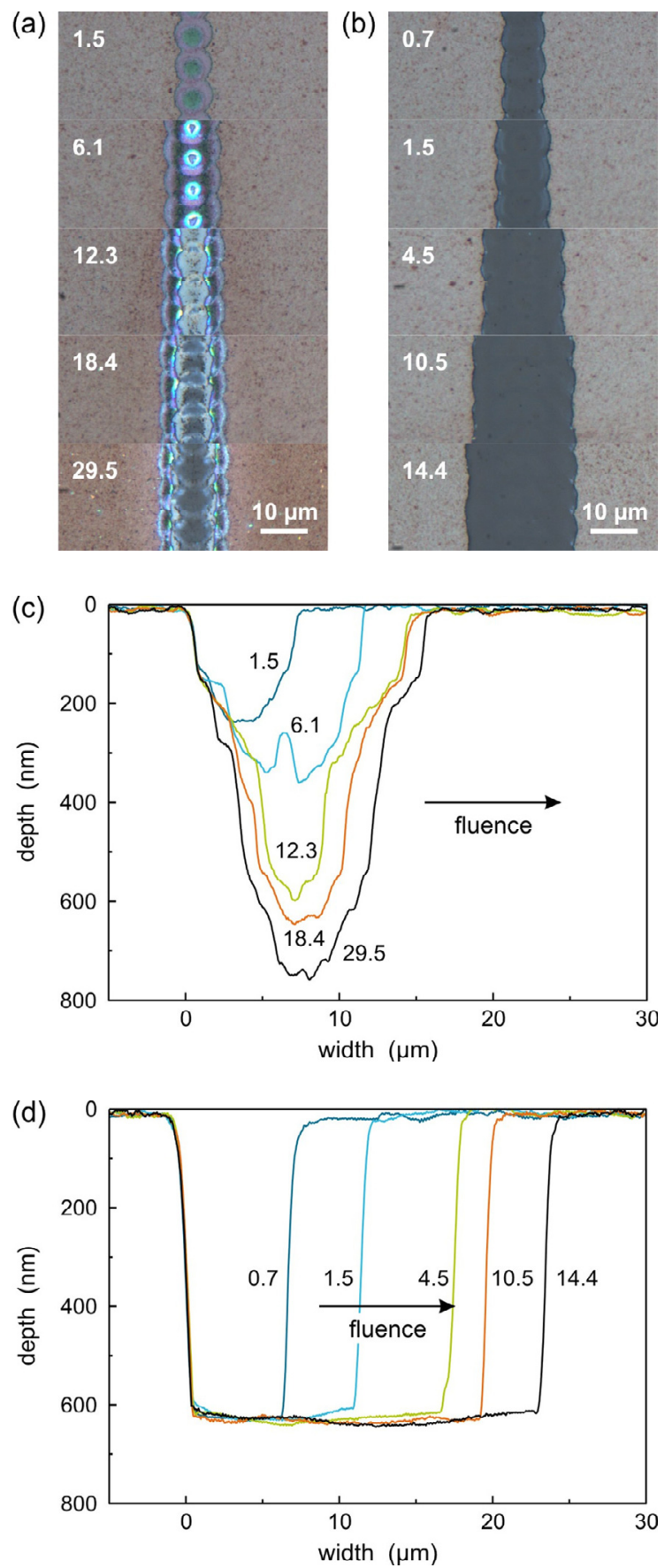

Fig. 2. Microscope images (a, b) and cross-sectional profiles $(c, d)$ of ablation lines; obtained after irradiation from the TCO side $(\mathrm{a}, \mathrm{c})$ or from the glass side (b, d). All numbers labeling the microscope images or depth profiles are fluence values in $\mathrm{J} / \mathrm{cm}^{2}$.

layer (light horizontal stripe in the center of the image) has clearly been delaminated from the glass substrate (lower region of the image), showing a dark gap of $b \approx 4 \mu \mathrm{m}$ width and maximum height of $\approx 60 \mathrm{~nm}$ in the middle of the irradiated area. Apparently, this is the region where the intensity of the Gaussian beam was above the threshold for fs laser-induced non-thermal evaporation, but the

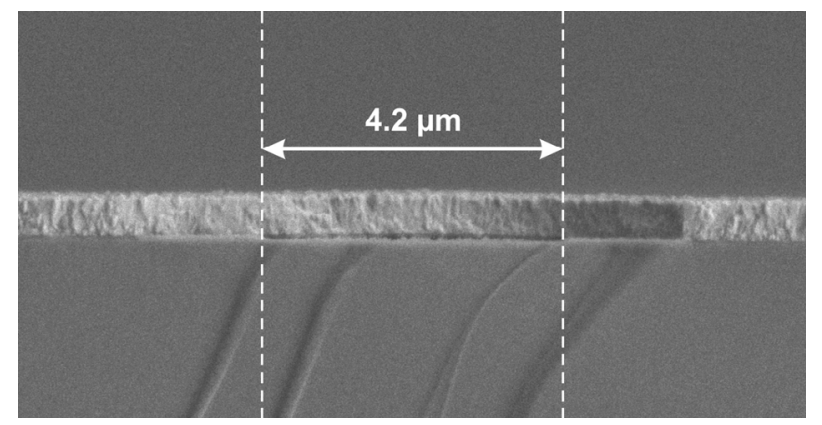

Fig. 3. SEM image of cross section through bulge after rearside irradiation slightly below ablation threshold.

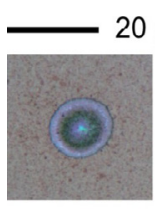

6.1

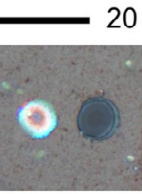

0.6 $\mu \mathrm{m}$

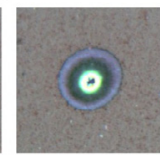

7.7

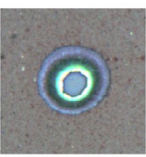

13.8

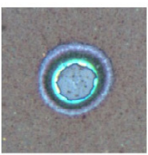

19.9

peak fluence $\left(\mathrm{J} / \mathrm{cm}^{2}\right)$

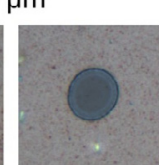

1.1

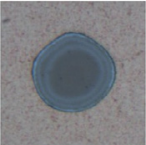

4.5

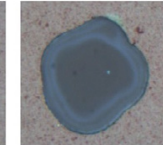

10.5

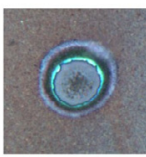

29.5 peak fluence $\left(\mathrm{J} / \mathrm{cm}^{2}\right)$

Fig. 4. Microscope images of single ablation spots. Upper row: irradiation from the TCO side; lower row: irradiation from the glass side.

resulting pressure at the interface was still not high enough to break and lift off the TCO layer. The layer thickness of evaporated material can only be estimated to be of the order of $10 \mathrm{~nm}$. Nevertheless this is a clear indication that the proposed mechanism has occurred in the ITO layer at the interface.

For a quantitative evaluation of the ablation thresholds another series of experiments was conducted with considerably increased spot-to-spot distance in order to exclude any possible effects due to interaction of two successive pulses. A selection of microscope images from this series obtained at different peak laser fluence from either side of the sample is collected in Figure 4. The images in the upper row of Figure 4 represent results of irradiation from the ITO side, while those in the bottom row have been obtained by sending the laser beam through the glass substrate first; the fluence increases from left to right in both cases. At the lowest fluence shown in the bottom row, some spots were not ablated, but only show a blister; here the fluence was apparently very close to ablation threshold, so that even the small laser fluctuations were sufficient to statistically lead to ITO ablation or only the above discussed blister formation. Therefore both effects were included in the bottom left image. 


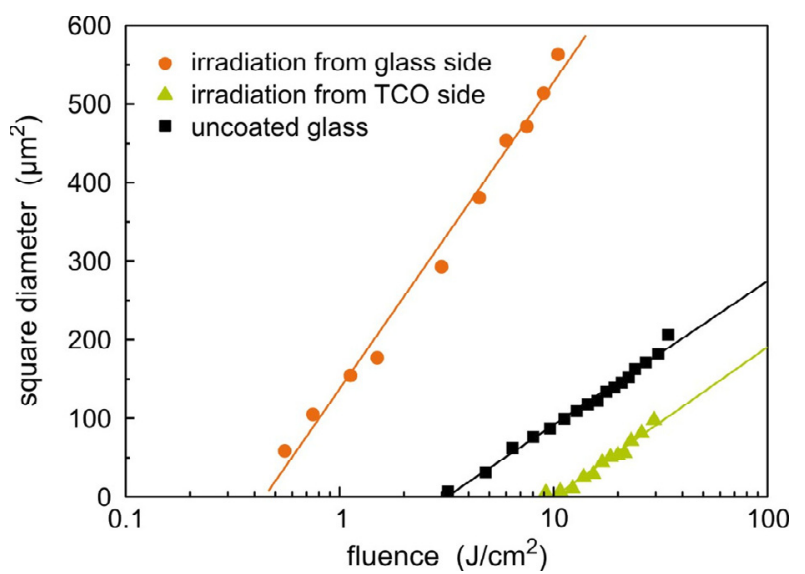

Fig. 5. Liu plot of squared diameters of ablation areas obtained by irradiation of TCO/glass samples from TCO (triangles) or glass side (circles), and of a pure glass substrate (squares) for comparison.

Apart from that, the images shown in Figure 4 indicate fairly well the same behavior as observed for considerable pulse overlap. Focusing on the difference between front-side and rear-side irradiation, it is noted that the rear-side illumination (lower panel in Fig. 4) creates in all cases craters with steep edges and a depth corresponding to the ITO thickness; the area of the opened glass surface increases monotonously with fluence. In contrast, irradiation from the layer side produces a trough of increasing depth first, before at higher fluence within this trough a smaller bulge with a narrow hole in the center occurs (situation at $6.1 \mathrm{~J} / \mathrm{cm}^{2}$, top left image). With further increase of fluence, the diameter of this central hole increases, then the crater bottom in the center reaches the glass (image at $13.8 \mathrm{~J} / \mathrm{cm}^{2}$ ); finally, upon further increase of laser pulse fluence, the opened glass surface area increases on expense of the surrounding rings, whereas the outer rim of the color change remains almost constant.

To determine the ablation thresholds for the complete series of ablation spots, the relevant areas (typically the squared diameter in case of a circular spot) have to be plotted versus laser pulse fluence on a logarithmic scale; then the ablation thresholds $\Phi_{\mathrm{A}}$ can be derived by extrapolating to zero ablation area. The relevant diameters are clearly the sharp crater edges for the rear-side irradiation as well as for another series of ablation experiments on the pure glass substrate which has been performed for comparison. In case of the ITO-side irradiation, the relatively steep walls of the inner crater (the narrow hole occurring around $6 \mathrm{~J} / \mathrm{cm}^{2}$ ) were selected, because this is apparently the process which at higher fluence leads to complete ablation of the ITO layer. The results of this analysis are plotted in Figure 5. It is obvious that $\Phi_{\mathrm{A}}$ for removing ITO is more than an order of magnitude lower for irradiation from the rear (glass) side $\left(\approx 0.5 \mathrm{~J} / \mathrm{cm}^{2}\right.$ and $\approx 9 \mathrm{~J} / \mathrm{cm}^{2}$, respectively). Apparently, a peak pulse fluence which does not cause visible changes when the ITO surface is irradiated causes already ablation of a small disk of the whole ITO layer when the same laser pulse enters through the glass substrate. Removal of the whole layer by irradiation from the ITO side, on the other hand, consumes so much laser energy for evaporation of the material that the respective $\Phi_{\mathrm{A}}$ is even higher than the ablation threshold for pure glass $\left(\approx 3 \mathrm{~J} / \mathrm{cm}^{2}\right)$

All these observations confirm the above proposed mechanism for ITO removal upon femtosecond irradiation through the substrate glass: apparently, the fs laser pulse creates via the existing free carrier absorption a critical carrier density $[12,13]$ in an ultrathin ITO layer adjacent to the ITO/glass interface, which leads to (non-thermal) evaporation of this layer, and the resulting high pressure breaks the ITO layer and leads to blasting off a disk, the outer rim of which is defined by the ablation threshold being reached within the Gaussian beam.

Irradiation from the ITO layer side will of course initiate the same mechanism. However, this means that comparable fluence can only ablate the ultra-thin layer having very high carrier density, because the resulting metal-like absorption will shield the deeper regions of the ITO layer from the highest pulse intensity. Therefore, higher fluence can only successively lead to deeper ablation, and since the pulse energy must be sufficient to evaporate the whole ITO material to be removed, the ablation threshold to achieve $d \approx 600 \mathrm{~nm}$ is more than one order of magnitude above that for rear-side processing. This is clearly a disadvantage in view of technological applications, as is the rather irregular, non-rectangular profile of the grooves. In contrast, the almost rectangular profile and the low ablation threshold found for rear-side illumination makes this approach very attractive for micro-structuring applications of ITO and other transparent conductive oxide films on glass substrate.

The only open point to discuss here is the observation that the groove bottoms are not perfectly flat, but turn out to be 20-30 nm deeper in the groove center in all cases (see Fig. 2); in the microscope images these central areas appear dark grey, while the outer regions of grooves or craters exhibit a bluish ring in most cases. This can be taken as hint that the center really represents a glass surface, while the outer ring might indicate a thin residue of a layer of different composition. A possible explanation for such a rest comes from the fact that the glass substrate has several constituents with high diffusion coefficients (like $\mathrm{Na}^{+}$or $\mathrm{K}^{+}$): during deposition of the ITO layer, inter-diffusion at the interface might create a transition zone from isolating glass to semiconducting ITO. Other than in the case of dielectric layers on silicon with an almost atomically sharp interface, this would cause a gradient of free carrier concentration and absorption over the transition zone. Under such circumstances, the critical carrier density required for the non-thermal ablation of the capping ITO layer may be achieved closer to the interface (original glass surface) at the highest pulse intensity in the center of the Gaussian beam profile, but in slightly larger distance in the outer regions. This could easily explain the shallow indentation observed in the laser trenches.

For technological applications, however, the indentation in the grooves is not a constraint, irrespective of its 
S. Krause et al.: Precise microstructuring of indium-tin oxide thin films on glass...

origin: preliminary conductivity measurements across the laser scribes showed that perfect electrical isolation (sheet resistance $\left.>10^{6} \Omega / \mathrm{sq}\right)$ is achieved even for the narrowest trenches (width $\approx 5 \mu \mathrm{m}$; see Fig. $2 \mathrm{~b}$ ) created by rear-side processing. More details on this point as well as further results for other TCO materials will be discussed in an extended study to be published separately.

\section{Summary}

In conclusion, single pulse femtosecond laser ablation of TCO (ITO) thin films from soda-lime glass substrates has been studied for irradiation both from the layer side and from the glass side. The rear-side illumination turned out to be a very effective tool to remove the ITO layer selectively and without noticeable thermal damage, enabling grooves with almost rectangular cross section, i.e. very steep edges and a flat bottom located at the initial position of the glass/TCO interface. The physical mechanism causing this fs laser lift-off process is apparently ultrafast non-thermal evaporation of a very thin TCO layer via laser-induced generation of a critical carrier density, in analogy to very recent results obtained on silicon wafers coated with thin dielectric layers. Technologically, the method studied in this work has a large potential for precise micro-structuring of TCO layers for various purposes. For thin film photovoltaics, the results presented here allow minimizing the area needed for the so-called P1 scribe (to produce electrically isolated TCO stripes), which is the first step towards the serial electrical connection of all the parallel stripes in a typical thin film solar cell. However, the applicability of this selective ablation technique is not limited to TCO, but can also be transferred to scribing of further layers of thin film solar cells, e.g. the P2 scribe to structure the absorber layer.
This work was supported by the FhG Internal Programs under Grant No. Attract 692 034. In addition, the authors would like to thank the German Federal Ministry for Education and Research ("Bundesministerium für Bildung und Forschung") for the financial support within the Centre for Innovation Competence SiLi-nano ${ }^{\circledR}$ (Förderkennzeichen: 03Z2HN11).

\section{References}

1. D. Ashkenasi, G. Müller, A. Rosenfeld, R. Stoian, I.V. Hertel, N.M. Bulgakova, E.E.B. Campbell, Appl. Phys. A 77, 223 (2003)

2. J. Hermann, M. Benfarah, G. Coustillier, S. Bruneau, E. Axente, J.-F. Guillemoles, M. Sentis, P. Alloncle, T. Itina, Appl. Surf. Sci. 252, 4814 (2006)

3. G. Raciukaitis, M. Brikas, M. Gedvilas, T. Rakickas, Appl. Surf. Sci. 253, 6570 (2007)

4. S. Zoppel, H. Huber, G. Reider, Appl. Phys. A 89, 161 (2007)

5. P. Gecys, G. Raciukaitis, M. Gedvilas, A. Selskis, Eur. Phys. J. Appl. Phys. 46, 12508 (2009)

6. A. Schoonderbeek, V. Schütz, O. Haupt, U. Stute, J. Laser Micro Nanoeng. 5, 248 (2010)

7. P. Gecys, G. Raciukaitis, M. Ehrhardt, K. Zimmer, M. Gedvilas, Appl. Phys. A 101, 373 (2010)

8. T. Rublack, S. Hartnauer, P. Kappe, C. Swiatkowski, G. Seifert, Appl. Phys. A 103, 43 (2011)

9. T. Rublack, M. Schade, M. Muchow, H.S. Leipner, G. Seifert, J. Appl. Phys. 112, 023521 (2012)

10. W. Wang, G. Jiang, X. Mei, K. Wang, J. Shao, C. Yang, Appl. Surf. Sci. 256, 3612 (2010)

11. J.M. Liu, Opt. Lett. 7, 196 (1982)

12. A. Cavalleri, K. Sokolowski-Tinten, J. Bialkowski, M. Schreiner, D. von der Linde, J. Appl. Phys. 85, 3301 (1999)

13. D. Arnold, E. Cartier, Phys. Rev. B 46, 15102 (1992)

Cite this article as: S. Krause, P.T. Miclea, F. Steudel, S. Schweizer, G. Seifert, Precise microstructuring of indium-tin oxide thin films on glass by selective femtosecond laser ablation, EPJ Photovoltaics 4, 40601 (2013). 